OPEN ACCESS

Edited by:

Hailan Liu,

Sichuan Agricultural University, China

Reviewed by:

Haiming $X u$,

Zhejiang University, China

Zaifeng $\mathrm{Li}$,

Hebei Agricultural University, China

${ }^{*}$ Correspondence:

Huihui Li

lihuihui@caas.cn:

h.li@cgiar.org

Dondrup Dawa

dwdunzhu@126.com

†These authors have contributed equally to this work

Specialty section:

This article was submitted to Statistical Genetics and Methodology,

a section of the journal

Frontiers in Genetics

Received: 09 March 2020

Accepted: 26 May 2020

Published: 03 July 2020

Citation:

Li Z, Lhundrup N, Guo G, Dol K, Chen P, Gao L, Chemi W, Zhang J, Wang J, Nyema T, Dawa D and Li H

(2020) Characterization of Genetic

Diversity and Genome-Wide

Association Mapping of Three

Agronomic Traits in Qingke Barley (Hordeum Vulgare L.) in the

Qinghai-Tibet Plateau.

Front. Genet. 11:638.

doi: 10.3389/fgene.2020.00638

\section{Characterization of Genetic Diversity and Genome-Wide Association Mapping of Three Agronomic Traits in Qingke Barley (Hordeum Vulgare L.) in the Qinghai-Tibet Plateau}

\author{
Zhiyong $\mathrm{Li}^{1+}$, Namgyal Lhundrup ${ }^{21}$, Ganggang Guo ${ }^{1}, \mathrm{Kar} \mathrm{Dol}^{3}$, Panpan Chen ${ }^{3}$, Liyun $\mathrm{Gao}^{2}$, \\ Wangmo Chemi ${ }^{2}$, Jing Zhang ${ }^{1}$, Jiankang Wang ${ }^{1}$, Tashi Nyema ${ }^{2}$, Dondrup Dawa ${ }^{2 \star}$ and \\ Huihui $L i^{1,4 *}$ \\ ${ }^{1}$ Institute of Crop Sciences, Chinese Academy of Agricultural Sciences, Beijing, China, ${ }^{2}$ State Key Laboratory of Hulless \\ Barley and Yak Germplasm Resources and Genetic Improvement, Tibet Academy of Agriculture and Animal Sciences, Lhasa, \\ China, ${ }^{3}$ Tibet Agricultural and Animal Husbandry College, Nyingchi, China, ${ }^{4}$ International Maize and Wheat Improvement \\ Center (CIMMYT), Texcoco, Mexico
}

Barley (Hordeum vulgare L.) is one of the most important cereal crops worldwide. In the Qinghai-Tibet Plateau, six-rowed hulless (or naked) barley, called "qingke" in Chinese or "nas" in Tibetan, is produced mainly in Tibet. The complexity of the environment in the Qinghai-Tibet Plateau has provided unique opportunities for research on the breeding and adaptability of qingke barley. However, the genetic architecture of many important agronomic traits for qingke barley remains elusive. Heading date $(H D)$, plant height $(P H)$, and spike length (SL) are three prominent agronomic traits in barley. Here, we used genome-wide association (GWAS) mapping and GWAS with eigenvector decomposition (EigenGWAS) to detect quantitative trait loci (QTL) and selective signatures for $\mathrm{HD}, \mathrm{PH}$, and SL in a collection of 308 qingke barley accessions. The accessions were genotyped using a newly-developed, proprietary genotyping-by-sequencing (tGBS) technology, that yielded 14,970 high quality single nucleotide polymorphisms (SNPs). We found that the number of SNPs was higher in the varieties than in the landraces, which suggested that Tibetan varieties and varieties in the Tibetan area may have originated from different landraces in different areas. We have identified 62 QTLs associated with three important traits, and the observed phenotypic variation is well-explained by the identified QTLs. We mapped 114 known genes that include, but are not limited to, vernalization, and photoperiod genes. We found that $83.87 \%$ of the identified QTLs are located in the non-coding regulatory regions of annotated barley genes. Forty-eight of the QTLs are first reported here, 28 QTLs have pleotropic effects, and three QTL are located in the regions of the well-characterized genes HVVRN1, HvVRN3, and PpD-H2. EigenGWAS analysis revealed that multiple heading-date-related loci bear signatures of selection. Our results confirm that the barley panel used in this study is highly diverse, and showed a great promise for identifying the genetic basis of adaptive traits. This study should increase our understanding of complex traits in qingke barley, and should facilitate genome-assisted breeding for qingke barley improvement.

Keywords: qingke barley, genetic diversity, GWAS, EigenGWAS, adaptation 


\section{INTRODUCTION}

Barley (Hordeum vulgare L.) was domesticated in Israel and Jordan in the southern part of the Fertile Crescent approximately 10,000 years ago (Badr et al., 2000). With an average world production of $120 \mathrm{Mt}$ annually (Ullrich, 2010), barley ranks fourth among the most important cereal crops in the world (http://faostat.fao.org). Barley is mainly used for food, fodder, alcoholic beverage ingredient, and is generally considered to be a healthful food (Blake et al., 2010; Collins et al., 2010). In Qinghai-Tibet Plateau, six-rowed hulless (or naked) barley, called "qingke" in Chinese or "nas" in Tibetan, is mainly produced in Tibet, and Qinghai, Sichuan, and Yunnan provinces of China. In the Qinghai-Tibet plateau, Tibetans use qingke barley to make wine and for consumption (Tashi et al., 2013). As the main food of Tibetans, qingke barley has been grown on the QinghaiTibet Plateau for at least 3,500 years, most probably following its introduction via northern Pakistan, India and Nepal (Zeng et al., 2018). Tibetans have a rich spiritual and cultural connection to qingke barley on the Qinghai-Tibet Plateau due to its wide range of medicinal and nutritional uses. Therefore, analysis of the genetic diversity present in cultivated varieties of qingke barley is especially important.

The adaptation to diverse, high elevation environments makes qingke barley a unique resource for genetic study and barley breeding (Zeng et al., 2015). At present, the genetic architecture of grain starch quality (Li et al., 2014) and drought stress tolerance (Zeng et al., 2016) has been studied in qingke barley, and salt and aluminum tolerance have been studied in Tibetan wild barley (Qiu et al., 2011; Wu et al., 2011; Cai et al., 2013). In other studies, diverse barley lines from different regions, including the US (Zhou and Steffenson, 2013; Genievskaya et al., 2018), Europe (Xu et al., 2018), and India (Visioni et al., 2018), were used to identify the genetic architecture of complex traits (heading time, number of kernels per spike, grain yield) and disease resistance (durable spot, stripe rust) in barley. Although some studies used worldwide collections of barley germplasm, few have included barley varieties from Tibet (Pasam et al., 2012; Gyawali et al., 2017). Over the past decade, studies in barley (Cuesta-Marcos et al., 2008), wheat (Kiseleva et al., 2016), and rice (Yan et al., 2011) have shown that variations in heading date (HD), plant height $(\mathrm{PH})$, and spike length (SL) contribute to environmental adaptation in cereal crops and also influence grain yield. In earlier studies, biparental mapping populations were used to reliably detect QTL for $\mathrm{HD}, \mathrm{PH}$, and spike morphological traits (Lin et al., 1998; Sameri et al., 2006; Zhang et al., 2009). With the emergence of more cost-effective, high-throughput genotyping technologies, single nucleotide polymorphisms (SNPs) related to HD have been identified by genome-wide association studies (GWAS) (Pasam et al., 2012; Visioni et al., 2013; Genievskaya et al., 2018), PH (Alqudah et al., 2016; Almerekova et al., 2019), leaf area (Alqudah et al., 2018), spike architecture (Comadran et al., 2011) and grain yield (Ingvordsen et al., 2015; Xu et al., 2018) in barley. However, the genetic study of complex agronomic traits in qingke barley is limited (Zhang et al., 2019).
For HD, important genes have been successfully isolated and characterized in barley. Exposure to low temperatures is known as vernalization, which is related to annual differences in seed production and flowering. This process protects the flowering meristem, which is sensitive to the cold, during winter (Yan et al., 2003; Trevaskis et al., 2006). Three genes control the vernalization parameters and growth conditions of barley: $H v V R N 1, H v V R N 2$, and HvVRN3. These are found on the respective chromosome arms $5 \mathrm{HL}, 4 \mathrm{HL}$, and $7 \mathrm{HS}$, all of which have been isolated (Laurie et al., 1995; Yan et al., 2003, 2004, 2006). A MADS-box transcription factor (TF) is encoded by $H v V R N 1$, which shares homology with APETALA1, CAULIFLOWER, and FRUITFULL. These are transcription factors that promote flowering in the apical meristem of Arabidopsis (Trevaskis et al., 2003; Yan et al., 2003; Trevaskis, 2010). A transcription factor with a zinc finger-CCT domain is encoded by $H v V R N 2$. While Arabidopsis has no homologous gene, its function is similar to FLOWERING LOCUS C (FLC), which inhibits flowering (Yan et al., 2004). FLOWERING LOCUS $T$ (FT) in Arabidopsis is similar to HvVRN3 in that it induces the expression of $H v V R N 1$ during periods of long daylight, promoting flowering (Yan et al., 2006; Distelfeld et al., 2009). In barley and wheat, $H v V R N 3$ integrates the photoperiod and vernalization pathways (Distelfeld et al., 2009). Another important pathway is that of the photoperiod, which regulates the date of flowering and heading and uses plant response daylight and optical cues from light receptors. It has been shown that $P p d-H 1$ is the ortholog of the wheat $P p d-D 1$ gene, a member of the pseudoresponse regulator $(P R R)$ gene family via homologybased cloning (Beales et al., 2007). The major determinants of the long-day response in barley are the Photoperiod-H1 (PpdH1) and Photoperiod-H2 ( $\mathrm{Ppd}-\mathrm{H} 2)$ genes on chromosomes $2 \mathrm{H}$ and $1 \mathrm{H}$, respectively (Abdullaev et al., 2017). The results of the study of Turner et al. (2005) suggest that Ppd-H1 might affect flowering by altering the expression of photoperiod pathway genes that are under circadian control. The dominant allele of $\mathrm{Ppd}-\mathrm{H} 1$ regulates response to increased photoperiod length and premature earing during long days. The recessive allele $p p d-$ $\mathrm{H} 1$ induces delays in heading during long days, while $\mathrm{Ppd}-\mathrm{H} 2$, a dominant allele, quickens heading during short days. The recessive allele impedes it.

For $\mathrm{PH}$, semi-dwarf genes include $u z u 1$, ari-e, and $s d w 1$ genes are widely used in modern barley improvement (Kuczynska et al., 2013; Dockter and Hansson, 2015). The ari-e gene has served in European cultivars and been located on chromosome 5HL (Froster, 2001). The $u z u$ gene, the primary dwarfing gene of East Asian barley strains, is located on chromosome 3HL (Zhang, 2000; Chono et al., 2003). Dwarfism regulated by $u z u$ is induced by the mutation of one nucleotide interchange in the $H v B R I 1$ gene, which involves brassinolide in the response (Chono et al., 2003). The chromosome 3HL is also the site of the $s d w 1$ gene, which is an important dwarfing gene in Europe, North America, South America, and Australia breeding programs (Jia et al., 2009; Xu et al., 2017). The dwarfism controlled by $s d w 1$ caused by a deletion mutation in the gibberellin 20-oxidase gene (HvGA20ox2) (Xu et al., 2017). Previous studies have shown that the QTLs controlling PH and SL are distributed on multiple 
TABLE 1 | Analysis of variance (ANOVA) of three traits across three locations.

\begin{tabular}{|c|c|c|c|c|c|c|}
\hline Trait & Source & $\mathrm{DF}^{\mathrm{e}}$ & Sum of square & Mean square & F-Value & $\operatorname{Pr}>F$ \\
\hline \multirow[t]{2}{*}{ HD_LS ${ }^{a}$} & Genotype & 307 & 22587.70 & 73.58 & 9.44 & 0.00 \\
\hline & Replicate & 2 & 5627.66 & 2813.83 & 361.03 & 0.00 \\
\hline \multirow[t]{2}{*}{$\mathrm{PH} \mathrm{NM}^{\mathrm{b}}$} & Genotype & 307 & 142700.59 & 464.82 & 8.32 & 0.00 \\
\hline & Replicate & 2 & 27.28 & 13.64 & 0.24 & 0.78 \\
\hline \multirow[t]{2}{*}{$S L \_N C^{C}$} & Genotype & 256 & 1016.82 & 3.97 & 5.04 & 0.00 \\
\hline & Replicate & 2 & 6.21 & 3.11 & 3.95 & 0.02 \\
\hline \multirow[t]{2}{*}{ SL_NM ${ }^{d}$} & Genotype & 307 & 1010.40 & 3.29 & 3.45 & 0.00 \\
\hline & Replicate & 2 & 5.26 & 2.63 & 2.76 & 0.06 \\
\hline
\end{tabular}

${ }^{a} H D_{L} L S$, heading date in Lhasa.

${ }^{b} \mathrm{PH} \_N M$, plant height in Namling.

cSL_NC, spike length in Nyingchi.

dSL_NM, spike length in Namling.

${ }^{e} D F$, degree of freedom.

chromosomes (Gyenis et al., 2007; Pasam et al., 2012; Fakheri et al., 2018), and that QTLs for PH and SL are identified on different chromosomes in different environments and treatments (Gyenis et al., 2007; Fakheri et al., 2018). In a wild x cultivated barley cross, Gyenis et al. (2007) identified QTLs for PH on chromosomes $1 \mathrm{H}, 2 \mathrm{H}, 3 \mathrm{H}$, and $7 \mathrm{H}$, and for SL on chromosomes $1 \mathrm{H}, 2 \mathrm{H}, 3 \mathrm{H}$, and $6 \mathrm{H}$. Another study identified QTLs for $\mathrm{PH}$ on chromosomes $2 \mathrm{H}, 3 \mathrm{H}, 4 \mathrm{H}, 5 \mathrm{H}, 6 \mathrm{H}$, and $7 \mathrm{H}$ in a spring barley collection (Pasam et al., 2012). A recent study suggests that QTLs for $\mathrm{PH}$ are distributed on chromosomes $5 \mathrm{H}$ and $7 \mathrm{H}$ and for SL on chromosomes $1 \mathrm{H}, 2 \mathrm{H}, 5 \mathrm{H}$, and $6 \mathrm{H}$ in Western European barley cultivars exposed to drought (Fakheri et al., 2018).

As the growth range of barley increased, it adapted to a wide spectrum of agricultural conditions. Studying selection signals in the barley genome is important to help us understand how this genome reacted to the various agricultural conditions experienced during domestication (Russell et al., 2016). Zeng et al. (2015) resequenced the genomes of 10 Tibetan wild barley accessions to uncover patterns of adaptation to the stressful environment of the Tibetan plateau. Further resequencing of 177 Tibetan barley genomes was performed to better understand the selection markers for the adaptation of local highland barley in the exome capture target range of the genome using the fixation index $\left(F_{\mathrm{ST}}\right)$ approach (Zeng et al., 2018). Eight regions as possible selective regions were identified, including the location near the Naked caryopsis ( $n u d$ ) on chromosome 7H. Recently, EigenGWAS, which combines the statistical framework of GWAS with eigenvector decomposition, is a novel approach for identifying regions of the genome under selection in any genetic data where the underlying population structure is unknown. EigenGWAS has been applied to studies in evolution, ecology, breeding, and human genetics (https://github.com/gc5k/GEAR/ wiki/EigenGWAS).

In the present study, we collected old local qingke barley landraces in Tibet, modern qingke barley varieties, and representative qingke barley varieties from regions surrounding the Tibetan region. The genetic diversity was compared between the landraces and the two variety groups, and the trends in the changes in genetic structure from the landraces to the breeding varieties was considered. In this study, therefore, our objectives were to use the 14,970 high quality SNPs discovered using genotyping-by-sequencing (tGBS) in 308 qingke barley accessions to (1) understand the genetic diversity in the landraces and the modern varieties and the changes in population structure that occurred going from the landraces to the breeding varieties, (2) identify genetic loci associated with $\mathrm{HD}, \mathrm{PH}$, and SL by GWAS, and (3) identify loci that underwent selection for environmental adaptation using EigenGWAS. The findings of this study could facilitate a better understanding of the genetic mechanisms underlying the establishment of adaptive traits and genome-assisted selection in qingke barley breeding.

\section{MATERIALS AND METHODS}

\section{Plant Materials}

A total of 308 qingke barley accessions were used in this study; 206 qingke landraces, 72 qingke varieties, and 30 varieties (including 18, 5, 1, and 6 varieties from Qinghai, Gansu, Yunnan, and Sichuan provinces of China, respectively). All the 308 accessions were planted in Tibet at three locations; Lhasa $\left(\mathrm{N} 29^{\circ} 36^{\prime}, \mathrm{E} 1^{\circ} 06^{\prime}\right)$ in April 2018, Namling $\left(\mathrm{N} 29^{\circ} 18^{\prime}, \mathrm{E} 88^{\circ} 46^{\prime}\right)$ on May 2017, and Nyingchi (N29 $39^{\prime}$, E94 $\left.21^{\prime}\right)$ in October 2017 with three replicates each. We used a randomized design to construct the field experiment. At each location, 30 seeds of each accession were planted in a plot with two rows of $150 \mathrm{~cm}$ long and $30 \mathrm{~cm}$ between rows. HD was measured as the number of days when the head first emerged from the flag leaf sheath on the main shoot in a plot (Zadoks scale, $Z=50$; Hemming et al., 2009). The PH was measured as the above-ground plant height without the awns. The SL was measured as the length from the base of main spike to the tip of main spike (excluding awns). All traits were measured as the average of five random plants.

\section{Phenotypic Data Analysis}

The Pearson's correlation coefficients between the traits and the broad sense heritability $\left(H^{2}\right)$ of target traits were calculated by AOV functionality in QTL IciMapping v.4.1 (Meng et al., 2015). In the analysis of variance of the three traits, variance 


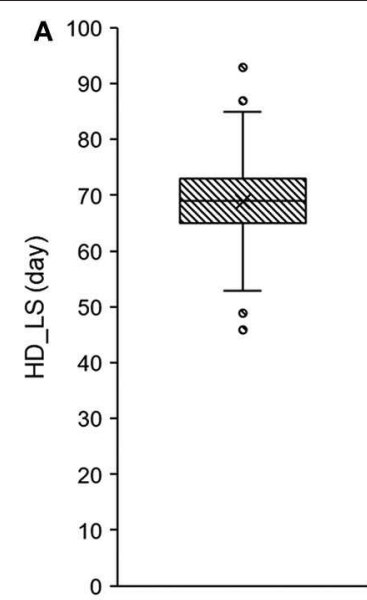

$H^{2}=73.99 \%$
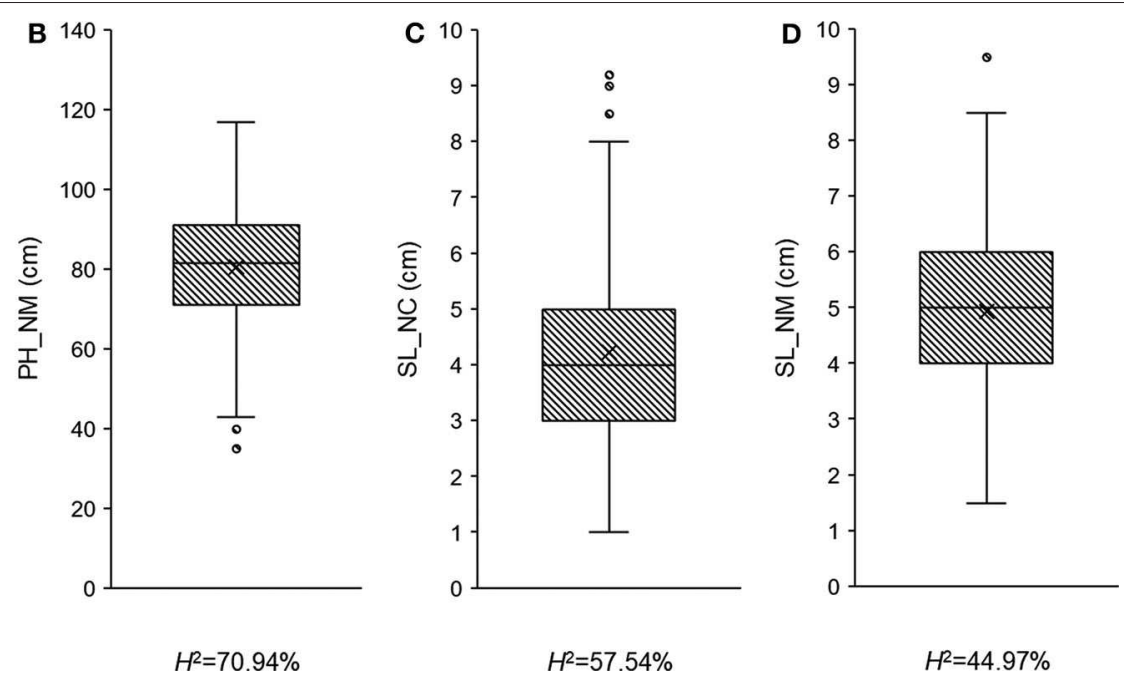

FIGURE 1 | The phenotypic distribution and heritability in broad sense for heading date in Lhasa (HD_LS) (A), plant height in Namling (PH_NM) (B), spike length in Nyingchi (SL_NC) (C), and spike length in Namling (SL_NM) (D).

components were estimated from a linear model; phenotype was partitioned into overall mean, genotypic effect, replication effect (i.e., location), and random error effect, all of which were treated as fixed effects. The $H^{2}$ on plot level was estimated from the following equation:

$$
H^{2}=\frac{\sigma_{G}^{2}}{\sigma_{G}^{2}+\sigma_{\varepsilon}^{2}},
$$

where $\sigma_{G}^{2}$ is the genetic variance and $\sigma_{\varepsilon}^{2}$ is the variance of the error. Although 308 accessions were planted at three locations, $\mathrm{HD}, \mathrm{PH}$, and SL were not all measured in three locations. Only SL has high-quality data in two locations (Nyingchi and Namling) in Tibet, abbreviated as SL_NC and SL_NM, respectively. For $\mathrm{HD}$ ad $\mathrm{PH}$, phenotype from one location was used, $\mathrm{HD}$ in Lhasa (abbreviated as HD_LS) and $\mathrm{PH}$ in Namling (abbreviated as $\mathrm{PH} \_\mathrm{NM}$ ), since from other two locations either the $H^{2}$ were lower than $30 \%$, or only one measurement was available for each plant. Considering data with low heritability was not reliable to conduct GWAS, and data with no replication could not be used to estimate the $H^{2}$ and evaluate the data quality, we discarded the low-quality data. For clarity, HD_LS, PH_NM, SL_NC, and SL_NM were used in the following-up analysis.

\section{SNP Genotyping and Genotypic Data Analyses}

The 308 accessions were genotyped using a newly developed genotyping-by-sequencing technology (tGBS) that eases the process of sorting high-quality GBS sequencing libraries and results in more accurate SNP calling (Ott et al., 2017; Li et al., 2019). Sequence reads were aligned to the Hordeum vulgare Hv IBSC PGSB v2 reference genome (Mascher et al., 2017) after de-barcoding and trimming. SNP calling was conducted using only those reads that aligned to a single location in the reference genome. In total, 46,034 polymorphic sites for each accession were discovered, and the data was filtered as follows: missing values $\leq 0.4$, heterozygosity rate (Het. Rate) $\leq 0.2$, and minor allele frequency (MAF) $\geq 0.05$ (Supplementary Table 1). After filtering, 14,970 high-quality SNPs were retained in the follow-up analysis. To assess population diversity, genome-wide pairwise linkage disequilibrium (LD) was calculated between SNP pairs to investigate the potential of the array to capture all significant regions associated with the observed phenotypes using the software package TASSEL v5.2 (Bradbury et al., 2007). LD was estimated by using the squared allele-frequency correlation $\left(r^{2}\right.$; Weir and Cockerham, 1996) for pairs of loci, since $r^{2}$ is affected not only by recombination frequencies at the two sites, but also by the differences in allele frequencies between sites. Decay of LD was evaluated, as was the distance between sites in base pairs (bp) with non-linear regression as implemented in the $\mathrm{R}$ package (Remington et al., 2001). To avoid multiple significances within individual LD blocks, the support interval was determined when the decay distance of LD reached $r^{2}=0.5$. Nucleotide diversity $(\pi)$ across the barley genome was calculated with TASSEL v5.2. The population structure of the 308 accessions was evaluated using principle component analysis (PCA) and a phylogenetic tree. Pairwise distances were estimated between genotyped individuals using an unbiased model of substitution frequencies. Distance estimates were then used to construct a phylogenetic tree using the Neighbor-Joining-like algorithm described by Saitou and Nei (1987) and implemented in the NJS module of the APE R package (Paradis et al., 2004). Unlike conventional neighborjoining methods, the NJS algorithm is tolerant of missing data, enabling its use with GBS data. Relative branch lengths are proportional to the amount of divergence observed between individuals. The effective sample size was calculated according to the method in Powell et al. (2010) as implemented in the software GEAR. 

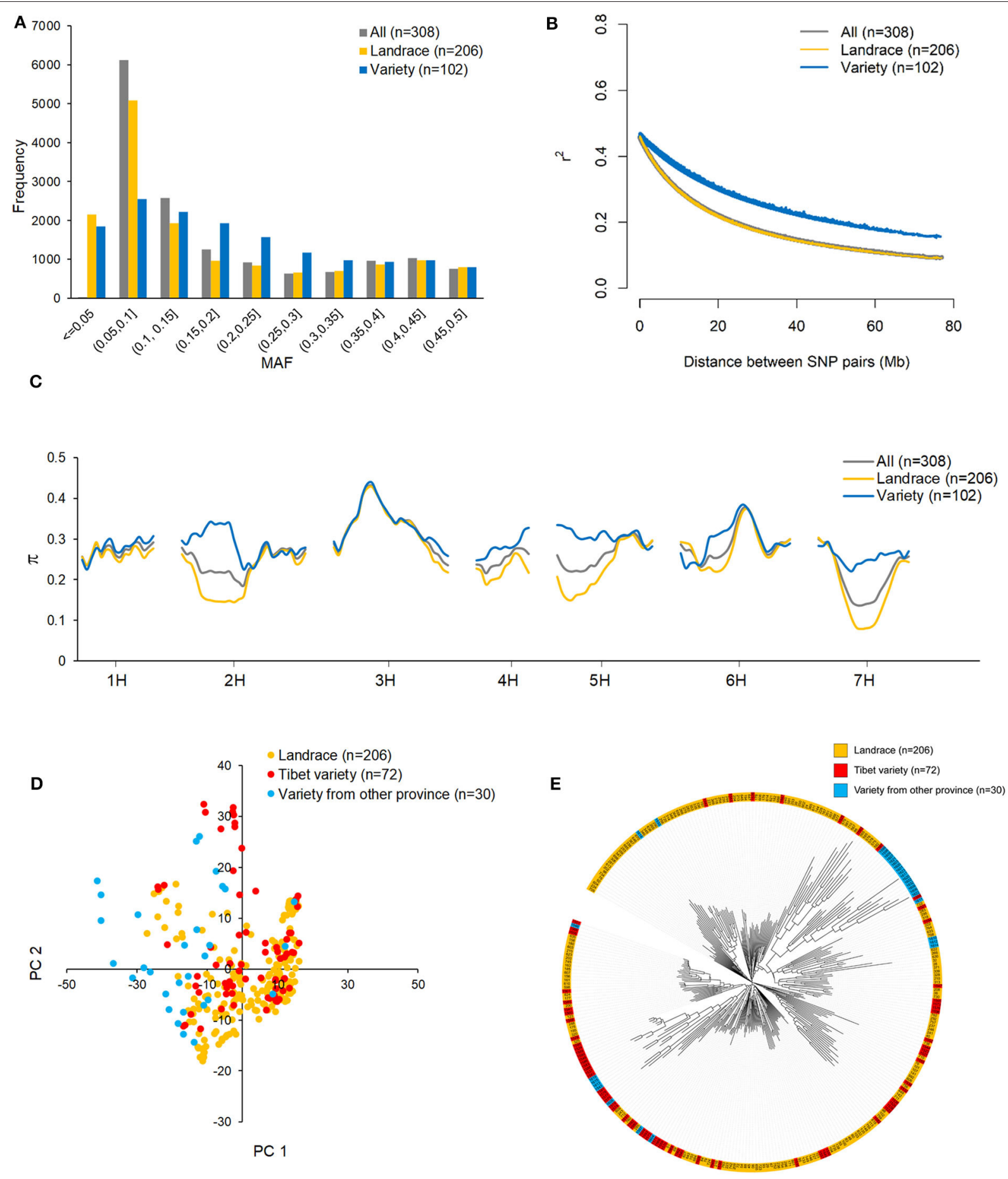

FIGURE 2 | The distribution of minor allele frequency (MAF) (A), linkage disequilibrium (LD) decay (B), and nucleotide diversity ( $\pi$ ) (C) across the barley genome in all 308 highland barley accessions, 206 landraces, and 102 varieties; the population structure of 308 barley accessions evaluated by principle component analysis (PCA) (D) and phylogenetic tree (E) base on 14970 high-quality SNPS.

\section{GWAS Analysis}

A GWAS for the three agronomic traits was conducted with a general linear model (GLM) and a mixed linear model (MLM) as implemented in TASSEL v5.2 software (Bradbury et al., 2007). For both models, the first principal component of the PCA was fitted as the cofactor to exclude the effect of population structure. In MLM, a variance-covariance kinship matrix, as covariates to estimate the association between phenotypes and genotypes (Zhang et al., 2010), was also considered. To declare QTL from the GWAS results, the phenotypic observation of SL 

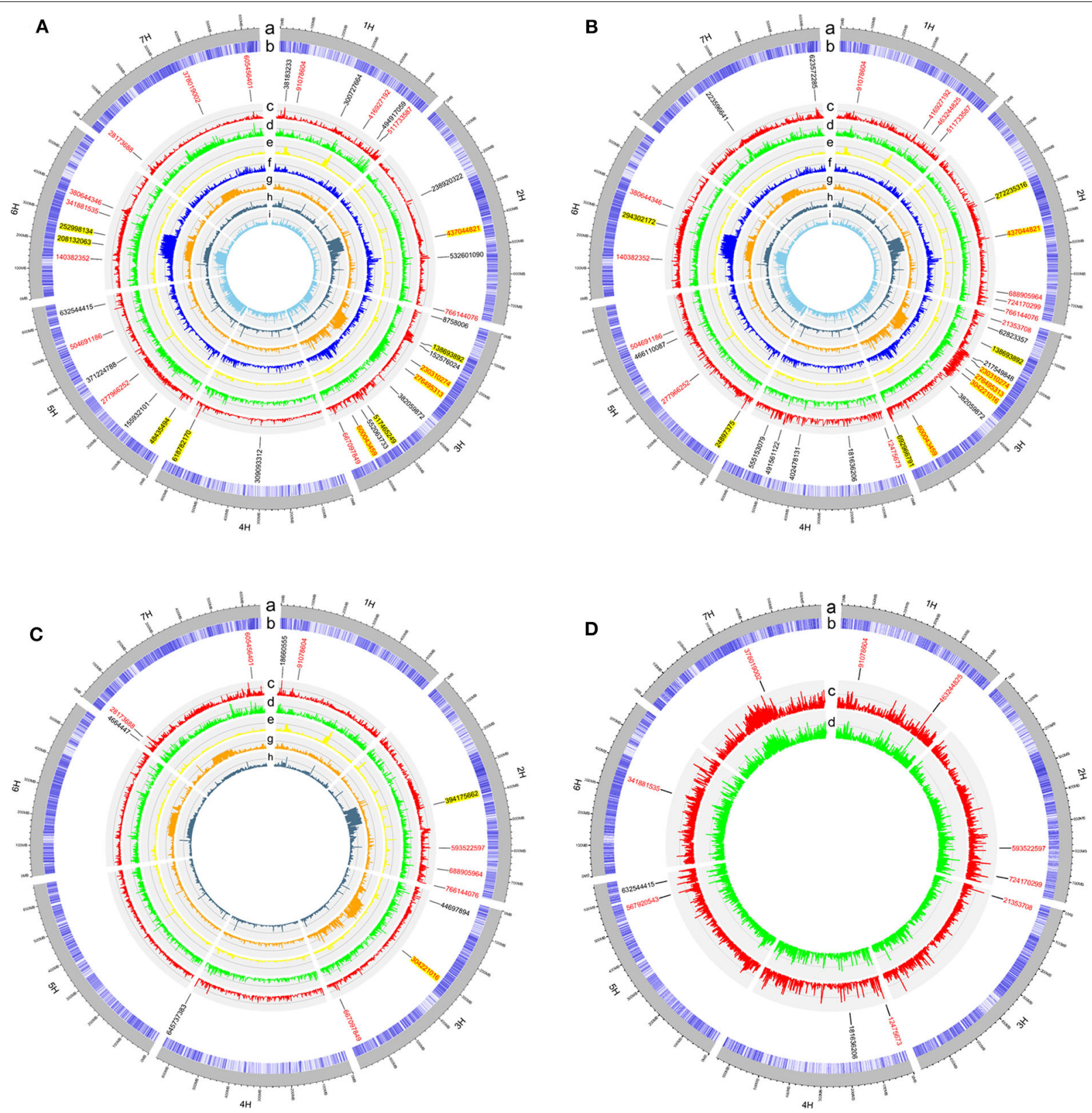

FIGURE 3 | The circular plots for heading date in Lhasa (HD_LS) (A), plant height in Namling (PH_NM) (B), spike length in Nyingchi (SL_NC) (C), and spike length in Namling (SL_NM) (D). From the outer circle to the inner circle, a is for the barley genome; $b$ is for the SNP density; $c$ is for the manhattan plot from generalized linear model (GLM); $d$ is for the manhattan plot from mixed linear model (MLM); $e$ is for the manhattan plot from EigenGWAS under the tenth eigenvector (EV10); $f$ is for the manhattan plot from EigenGWAS under the seventh eigenvector (EV7); $g$ is for the manhattan plot from EigenGWAS under the fifth eigenvector (EV5); $h$ is for the manhattan plot from EigenGWAS under the third eigenvector (EV3); and i is for the manhattan plot from EigenGWAS under the second eigenvector (EV2). The SNP positions associated with the trait of interest were marked in black font; of which with pleotropic effects were highlighted in red font; and detected by EigenGWAS were highlighted in yellow background.

was reshuffled 1,000 times to analyze the null distribution. We calculated the 95 th quantile of the 1,000 most significant $p$-values over 1,000 permutations to be 5.18 after $\log _{10}$ transformation. The Bonferroni correction, $-\log _{10}(1 / 14,970)=4.18$, was also calculated. To balance the false positives and false negatives, a $-\log _{10}(P)$ threshold of 4.00 was used for the GLM and 3.00 was used for the MLM. To determine whether the uncovered genetic architecture was appropriate, the identified QTL was used to predict the performance of the corresponding trait. The most significant SNP in each QTL region was fitted in the linear model with the original trait performance as the dependent variable. The adjusted coefficient of determination $\left(R^{2}\right)$ from the linear 
model was then calculated. The performance of QTL in different locations were estimated by $a=\frac{1}{e} \sum_{i=1}^{e} a_{i}$ and $a e_{i}=a_{i}-a$, where $a$ was the averaged effect of QTL across locations, $a_{i}$ was the additive effect of QTL for each location, $e$ is the number of locations, and $a e_{i}$ was the additive by environment effect of QTL in each location (Li et al., 2015).

\section{Analysis of Gene Annotation and Enrichment}

We used SnpEff to conduct functional annotations and effect predictions of the target SNPs (Cingolani et al., 2012). The Barley Hv_IBSC_PGSB_v2 reference genome gene annotation was downloaded as a gff3 file from the Ensembl plants database (http://plants.ensembl.org/index.html). Gene annotation information was acquired by BARLEX: The Barley Genome Explorer (https://apex.ipk-gatersleben.de/apex/f?p=284:10::.:::;; Colmsee et al., 2015). A Singular Enrichment Analysis (SEA) tool was used to perform a functional enrichment analysis of the annotated genes (Tian et al., 2017).

\section{EigenGWAS Analysis}

EigenGWAS is a regression approach based on principal component analysis (Chen et al., 2016; Li et al., 2019). It is similar to GWAS; however, the phenotype is replaced with an eigenvector (EV) to capture genetic variation in the studied population. In this study, EigenGWAS, implemented in the software GEAR (https:/github.com/gc5k/GEAR), was used to separate loci under selection by treating top 10 eigenvectors (i.e., EV1-EV10) as phenotypes. We adjusted the $p$-value using a genomic control factor, denoted as $P_{G C}$, to exclude the effect of the genetic drift (Devlin and Roeder, 1999), and used the $P_{G C}$ to identify loci under selection. We reshuffled the first eigenvector 1,000 times to identify the significance cutoff for the relevant loci, which helped us analyze the null distribution. We calculated the 95th quantile of the 1,000 most significant $p$-values over 1,000 permutations to be 5.75 after $\log _{10}$ transformation. Considering the Bonferroni correction 4.18 as mentioned above, a $-\log _{10}(P)$ threshold of 4.00 was applied for EigenGWAS analyses in all 10 eigenvectors.

\section{RESULTS}

\section{Phenotypic Variation and Correlation Analysis}

To determine whether the observed traits exhibit wide variation, are highly heritable, and/or display a normal distribution, the recorded phenotypic data was analyzed using ANOVA (Table 1 and Supplementary Table 2) and boxplots (Figure 1). Fifty-one plants had no measurement for SL_NC, so the degrees of freedom in this case was only 256 (Table 1). All the variance components were significant $(P<0.05)$ across trials, with the exception of the replicates in PH_NM and SL_NM (Table 1). Wide variations ranging from 46 to 93 days in HD_LS, from 35 to $117 \mathrm{~cm}$ in PH_NM, from 1 to $9.2 \mathrm{~cm}$ in SL_NC, and from 1.5 to $9.5 \mathrm{~cm}$ in SL_NM were observed in the collection of 308 qingke barley accessions (Figure 1). The SL distribution showed that the
SL_NM mean was higher than it was for SL_NC (Figures 1C,D), and the correlation between SL_NM and SL_NC was $0.21(P<$ 0.01; Supplementary Figure 1). The reason for this may be due to the big environmental difference between Namling $(4,000 \mathrm{~m}$ above sea level) and Nyingchi (2,995 $\mathrm{m}$ above sea level) and the overcast and rainy weather in Nyingchi at flowering time, which was not conducive to pollination and thus decreased the effective seed-setting rate of the barley spikes. The broad-sense heritabilities for the three observed traits ranged from 44.97 to 73.99\% (Figure 1). The highest correlation was between PH_NM and SL_NM (i.e., 0.48 with $P<0.01$; Supplementary Figure 1), and there was a negative correlation between SL_NC and HD_LS. These observations are consistent with the general experience regarding the relationships between $\mathrm{PH}$ and SL (Wang et al., 2010), and between SL_NC and HD_LS (Wang et al., 2010; Al-Tabbal and Al-Fraihat, 2012).

\section{Genetic Diversity and Population Structure in the 308 Qingke Barley Accessions}

The MAF distributions of all 14,970 SNPs in the whole dataset and in the landrace and variety subpopulations are shown in Figure 2A. Because the 14,970 SNPs were filtered to remove those with MAF $<0.05$ in the 308 accessions, the minimum MAF here is 0.05 , and the average MAF is 0.183 . The MAF ranged from 0 to 0.5 in both subpopulations. SNPs with MAF $<0.05$ were considered to be rare SNPs. In this sense, more rare SNPs were observed in the landrace subpopulation $(2,150)$ than in the variety subpopulation $(1,841)$. The numbers of SNPs with MAFs ranging from 0.05 to 0.1 were 5,086 and 2,545 in the landrace and variety subpopulations, respectively. This suggests that more low MAF SNPs are present in the landrace subpopulation than in the variety subpopulation. Non-linear models of LD decay for the 206 landraces and 102 varieties are shown in Figure 2B. In general, LD in both datasets showed an intermediate rate of decline. The predicted value of $r^{2}$ declined to 0.5 within $1 \mathrm{Mb}$, which is considered to be the length of the support interval. As expected, LD decayed faster in the landrace subpopulation than in the variety subpopulation. The predicted value of $r^{2}$ declined to 0.2 within $23 \mathrm{Mb}$ for the landraces and within $49 \mathrm{Mb}$ for the varieties. It remained $>0.1$ for over $80 \mathrm{Mb}$ in the varieties. Due to the different allele distribution of the SNPs in the two subpopulations, nucleotide diversity $(\pi)$ in the variety subpopulation was higher than in the landrace subpopulation, particularly on chromosomes $2 \mathrm{H}, 4 \mathrm{H}, 5 \mathrm{H}$, and 7H (Figure 2C).

To determine whether the population structure could be discerned from the whole-genome genotyping data, PCA (Figure 2D) and a phylogenetic analysis (Figure 2E) were conducted for the 308 accessions. Based on the PCA plot, the two subpopulations, landraces and varieties, could not be clearly separated. This is likely due to a large proportion of the varieties being derived from qingke barley landraces. However, from the phylogenetic tree, it cannot be ruled out that the 15 varieties from Gansu and Qinghai provinces were not derived from the Tibetan landraces (Figure 2E). 
TABLE 2 | QTL identified by GWAS using generalized linear model (GLM) and mixed linear model (MLM) and EigenGWAS.

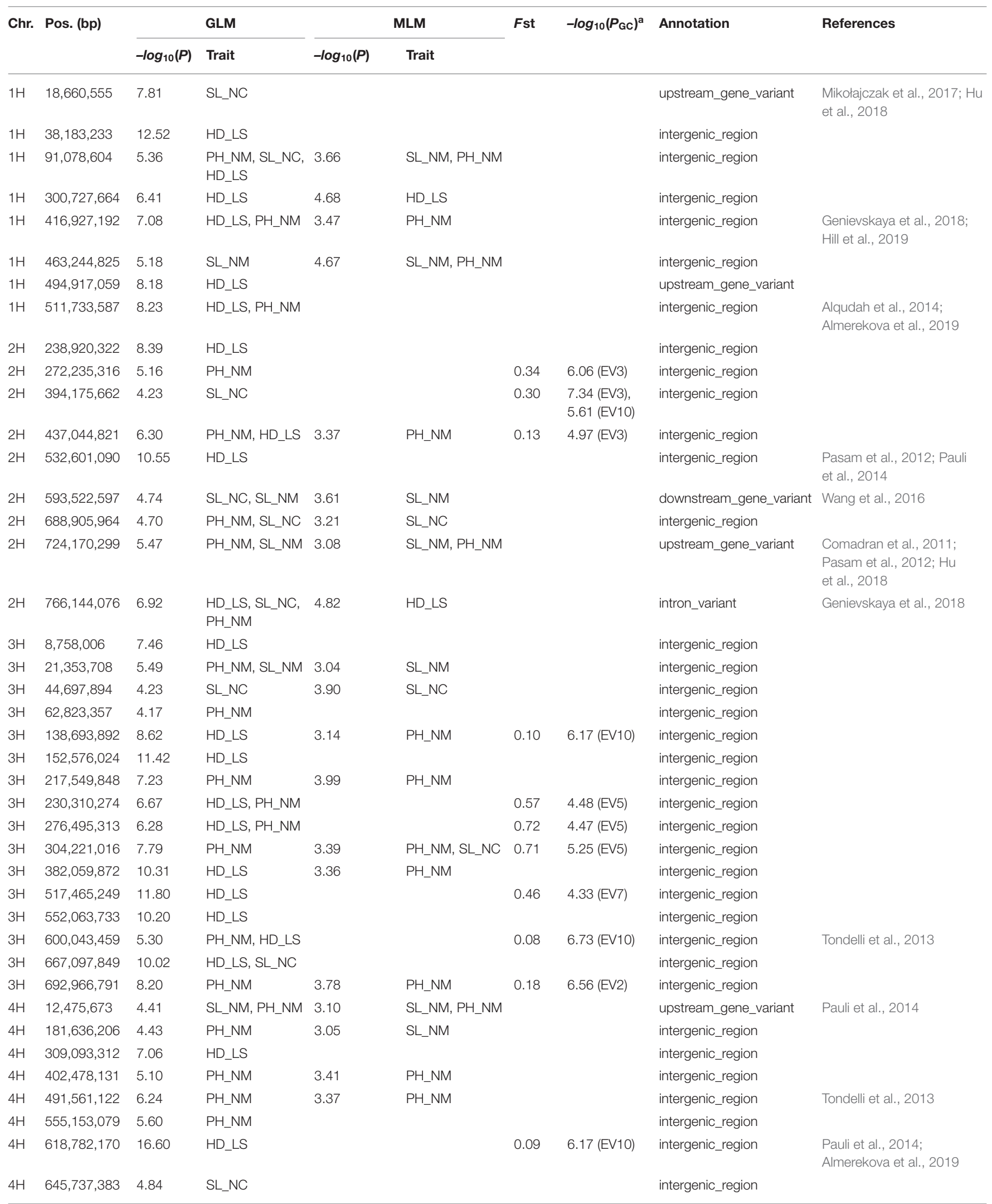


TABLE 2 | Continued

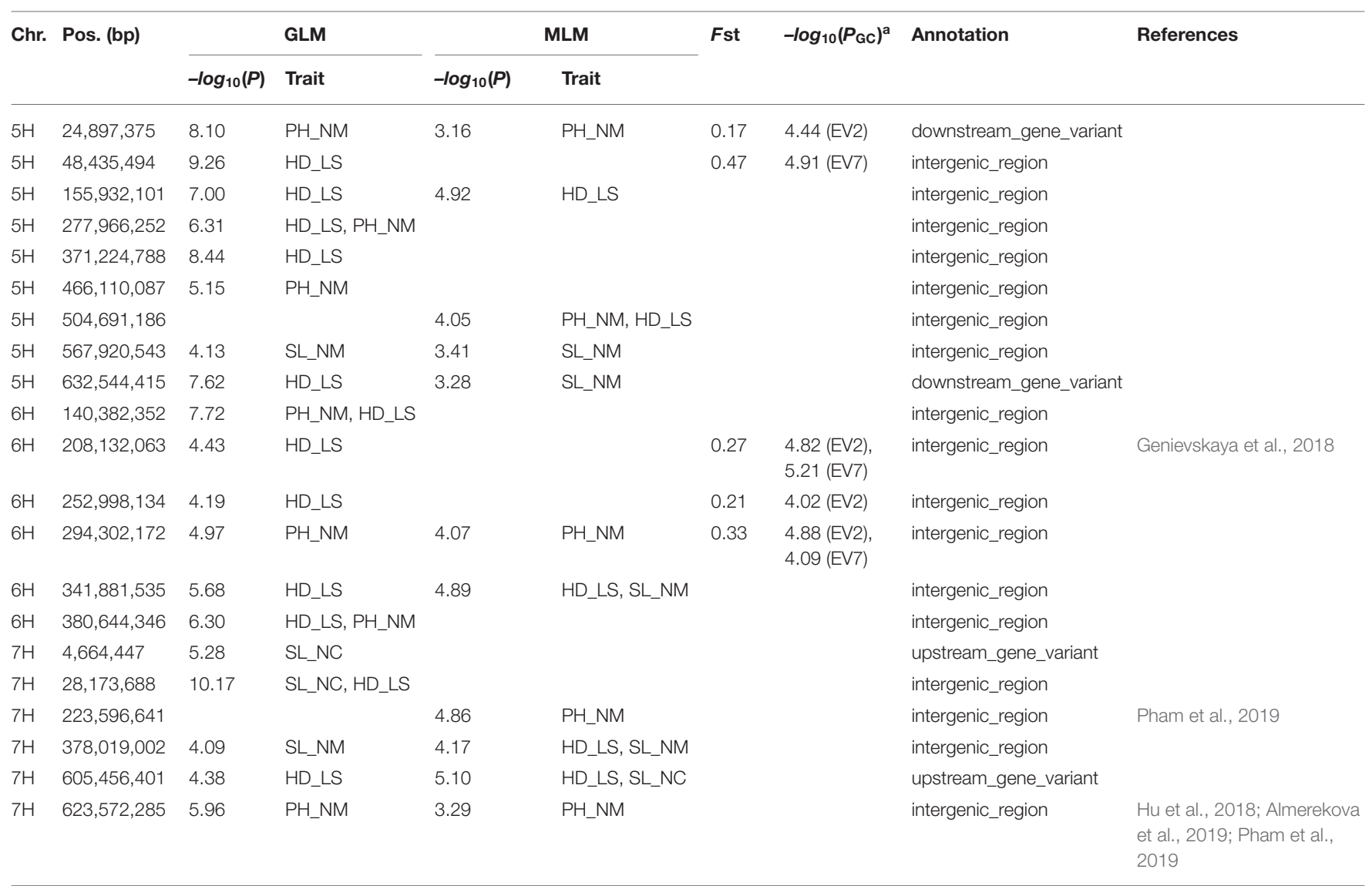

${ }^{a}$ Corrected p-value of EigenGWAS. Blank means the QTL was not identified by the corresponding method.

\section{GWAS and EigenGWAS}

In the GWAS, a total of 62 QTLs distributed across the barley genome that control three agronomic traits were identified either by GLM or by MLM (Figure 3 and Table 2). To evaluate if the first PC as cofactor was appropriate, no PC and PC number with 2-5 were also used to conduct GWAS (Supplementary Figures 2-11). Results showed that the parameter estimation would be inflated if no PC as cofactor in GWAS model. The parameter estimations from PC number 1-5 were fairly the same. Of the 62 QTLs, the largest number of QTLs, 16 , was distributed on chromosome $3 \mathrm{H}$, and the lowest number (6) was distributed on chromosomes $6 \mathrm{H}$ and $7 \mathrm{H}$. There were 29 QTLs (46.7\%) that were detected by both GLM and MLM; 16 QTLs were declared as selection loci by EigenGWAS under five eigenvectors (i.e., EV2, EV3, EV5, EV7, and EV10); six QTLs were reported by other studies, and six QTLs were consistently identified by GLM, MLM, and EigenGWAS (Table 2). In total, 28 QTLs had pleotropic effects (red text in Figures 3, 4A). One QTL with pleotropic effects located at $91,078,604 \mathrm{bp}$ on chromosome $1 \mathrm{H}$ was associated with all three traits in four trials. A QTL at 766,144,076 bp on chromosome $2 \mathrm{H}$ was related to the three traits HD_LS, PH_NM, and SL_NC, and was also associated with $\mathrm{PH}$, days to seed maturation (SMT), peduncle length (PL), and HD, as reported by Genievskaya et al. (2018).
Of 28 pleotropic-effect QTLs, six were detected by EigenGWAS as well, and these are shown in red text highlighted in yellow in Figures 3A-C and Table 2. These are the QTLs located at $437,044,821$ bp on chromosome $2 \mathrm{H}$ by EV3, $138,693,892 \mathrm{bp}$ on chromosome $3 \mathrm{H}$ by EV10, 230,310,274 bp on chromosome $3 \mathrm{H}$ by EV5, 276,495,313 bp on chromosome 3H by EV5, 304,221,016 bp on chromosome $3 \mathrm{H}$ by EV5, and 600,043,459 bp on chromosome $3 \mathrm{H}$ by EV10 (Figure 3 and Table 2). Two QTLs at 91,078,604 bp on chromosome $1 \mathrm{H}$ and at 593,522,597 bp on chromosome $2 \mathrm{H}$ associated with SL were both detected in two locations (Figures 3, 4A and Table 2).

In general, 36, 33, 12, and 11 QTLs were associated with HD_LS, PH_NM, SL_NC, and SL_NM, respectively (Figure 4B and Table 2), and are positively correlated with the broad sense heritabilities (Figure 1). In our study, we were able to investigate pleiotropy of QTLs on multiple traits. We observed that there were 16 QTLs for HD_LS and PH_NM in common. However, the correlation between HD_LS and PH_NM was not significant (Supplementary Figure 1), which may due to the repulsion linkage phase of the 16 QTLs (Supplementary Table 4). In contrast, six PH_NM QTLs were also significant for SL_NM, and the correlation between these two traits was 0.48 , which was highly significant. The reason for this may be that the six QTLs are in coupling linkage 

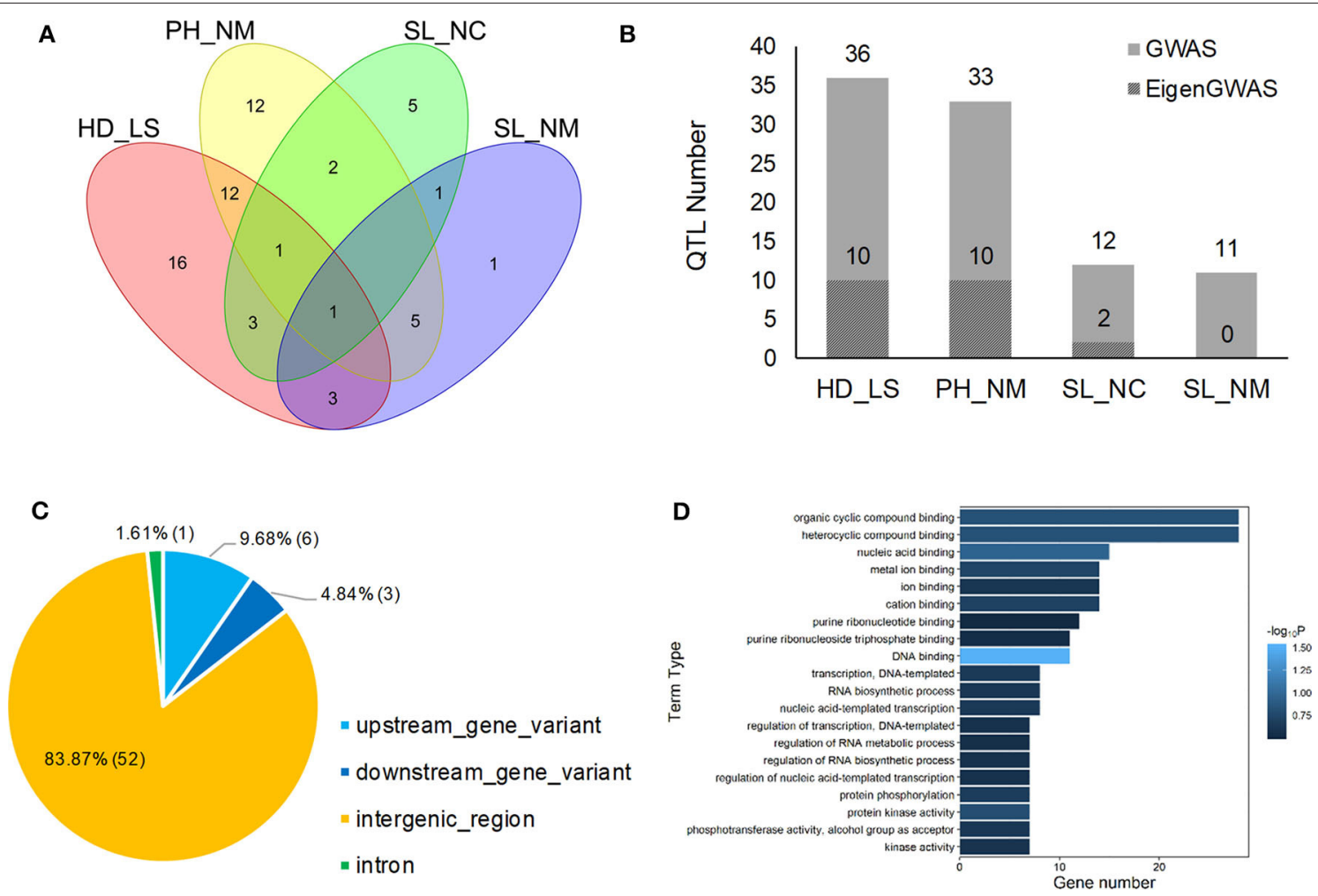

FIGURE 4 | Venn plot of QTL distribution for HD_LS, PH_NM, SL_NC, and SL_NM (A), the distribution of QTL number for heading date in Lhasa (HD_LS), plant height in Namling (PH_NM), spike length in Nyingchi (SL_NC), and spike length in Namling (SL_NM) (B); and gene annotation (C) and ontology (D) for the 62 QTL identified by GWAS.

phase (Supplementary Table 4). To evaluate the performance of QTL associated with SL in different locations, genotype by environment effects were estimated (Supplementary Figure 12 and Supplementary Table 5). The most significant genotype-byenvironment QTLs identified by both GLM and MLM were at $593,522,597 \mathrm{bp}$ on chromosome $2 \mathrm{H}$ and $21,353,708 \mathrm{bp}$ on chromosome $3 \mathrm{H}$, since their additive effects were both significant in Nyingchi, but not in Namling. In addition, there were three genotype-by-environment QTLs identified by GLM on chromosomes $1 \mathrm{H}, 2 \mathrm{H}$, and $7 \mathrm{H}$, respectively.

\section{Candidate Gene Annotation and Enrichment}

The annotation conducted on the 62 significant QTLs identified by GWAS (Table 2) showed that $52(83.87 \%)$ of QTL regions are intergenic, and that $10(16.13 \%)$ are genic (Figure $4 \mathrm{C}$ ). This is consistent with the Hordeum vulgare Hv IBSC PGSB v2 reference genome, where $19.2 \%$ of the barley genome is genic (Mascher et al., 2017) and a high ratio of loci $(78.00 \%)$ related to phenotypic variation are identified in intergenic regions (Mei et al., 2017). Of the QTL, 9.68\% and 4.84\% were located in the upstream and downstream gene regions, and $1.61 \%$ of the QTL were in the intron regions (Figure 4C). In total, 114 known genes were mapped as significant QTLs in the GWAS, and most of them were assigned to the "molecular function" and "biological process" categories in gene ontology (GO) analysis (Figure 4D). One QTL, located at 605,456,401 bp on chromosome 7H, controls HD_LS and SL_NC, and is $2.1 \mathrm{~Kb}$ upstream of HORVU7Hr1G100540, a known gene that encodes an SBP (S-ribonuclease binding protein) family protein. A QTL at $24,897,375$ on chromosome $5 \mathrm{H}$ significantly associated with PH_NM was found to be located $799 \mathrm{bp}$ downstream of the gene HORVU5Hr1G009980 that encodes a tetratricopeptide repeat (TPR)-like superfamily protein. For SL_NM and SL_NC, a stable QTL at $593,522,597 \mathrm{bp}$ on chromosome $2 \mathrm{H}$ is located 554 bp downstream of HORVU2Hr1G081800, which encodes a WPP domain interacting protein 2 (Supplementary Table 3 ). In addition, screening of the associated mapping population identified variations in $\mathrm{HD}$, and we found a significant $\mathrm{SNP}(5 \mathrm{H}$ : $599,361,872)$ near the vernalization gene $H v V R N 1$, a significant SNP (7H: 38,508,938) near the vernalization gene $H v V R N 3$, and a significant SNP $(1 \mathrm{H}: 514,145,049)$ near the photoperiod gene $\mathrm{PpD}-\mathrm{H} 2$.

\section{Phenotype Prediction}

To determine the accuracy of the QTL effect estimation, we used the significant QTL additive effect estimates to predict the phenotypic observations for the three traits, and were able to accurately predict HD_LS $\left(R^{2}=69.20 \%\right)$, PH_NM $\left(R^{2}=\right.$ $64.07 \%)$, and SL_NC $\left(R^{2}=42.37 \%\right)$ (Figure 5). For SL_NM, the prediction was low, due in part to the low heritability of SL in 


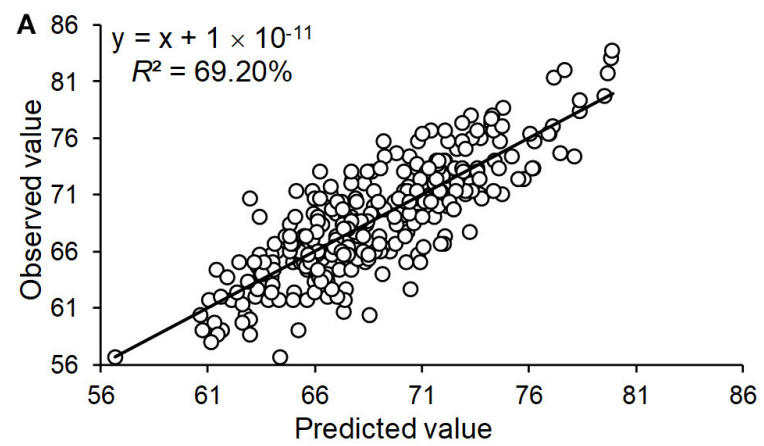

C

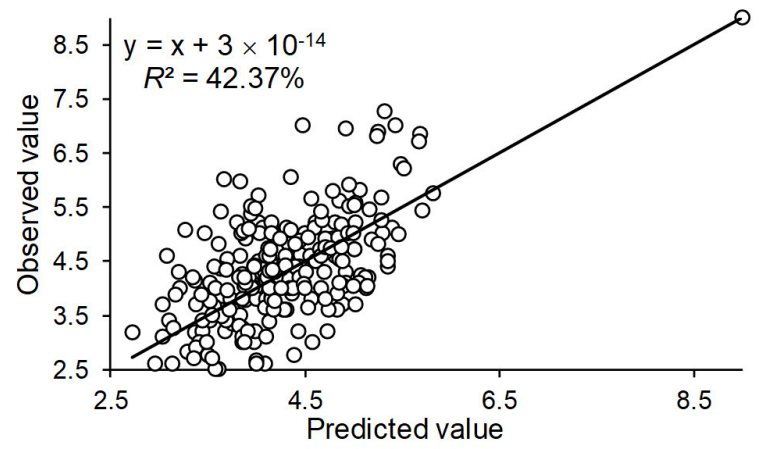

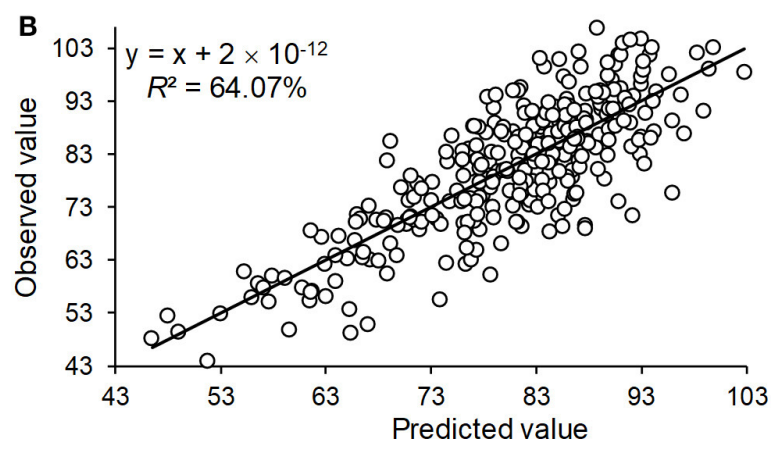

D

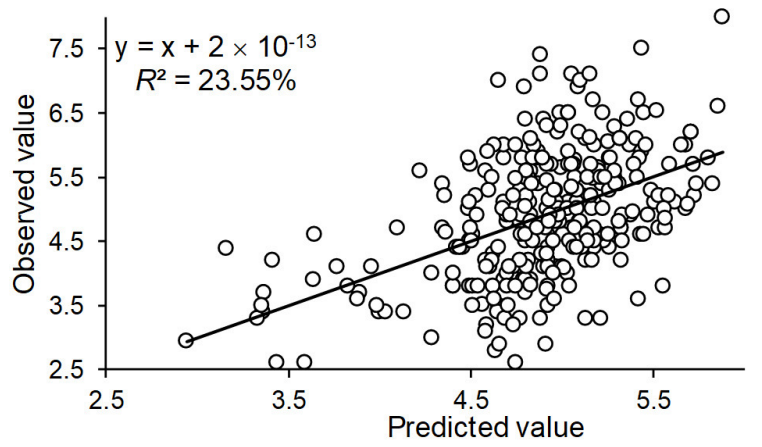

FIGURE 5 | The prediction of the observed phenotype by the QTL identified by GWAS for the traits of heading date in Lhasa (HD_LS) (A), plant height in Namling (PH_NM) (B), spike length in Nyingchi (SL_NC) (C), and spike length in Namling (SL_NM) (D).

NM (Figure 1). Looking at the broad sense heritabilities for the three traits (Figure 1) suggests that the QTL results presented in this study are reliable, and provide further evidence that a large proportion of the phenotypic variation can be explained by additive variance in this association panel.

\section{DISCUSSION}

To the best of our knowledge, few genetic studies have investigated the complex agronomic traits in Tibetan qingke barley (Zhang et al., 2019). Previous reports have included only a limited number of qingke barley accessions to identify potential signals of adaptation and domestication. For example, 95 wild barley accessions from Tibet and 28 six-rowed hulless barley varieties from Tibet and Xinjiang were used to show that the Tibetan Plateau and the surrounding areas are primary centers of barley cultivation (Dai et al., 2012); six wild-barley genotypes collected from the Tibetan Plateau were used in an RNA-seq analysis to reveal multiple origins of barley domestication (Dai et al., 2014); 10 Tibetan wild barley accessions were re-sequenced to uncover patterns of adaptation (Zeng et al., 2015); and 177 Tibetan barley accessions were re-sequenced to identify signals of selection in the genome (Zeng et al., 2018). In contrast, 308 qingke accessions from the Qinghai-Tibet Plateau, including 278 qinke barley accessions and 30 qingke varieties collected from five other Chinese provinces, were used for this study. The effective sample size is 272 in total, which is comprised of 182.63 in the landrace subpopulation and 89.37 in the variety subpopulation.
Our results demonstrate that this panel has a large effective population size with high levels of intra-species genetic flow, making it a suitable candidate for the characterization of genetic structure and adaptation, and was appropriate for the genetic study of complex traits by GWAS.

Previous studies have shown that QTLs identified on all seven chromosomes are significantly associated with HD, except for photoperiod and vernalization loci (Pasam et al., 2012). QTLs located on chromosomes $1 \mathrm{H}, 2 \mathrm{H}$, and $5 \mathrm{H}$ have been identified that are significantly associated with $\mathrm{PH}$ in a worldwide spring barley investigation (Alqudah et al., 2016). Another recent study shows that QTLs on chromosomes $5 \mathrm{H}$ and $7 \mathrm{H}$ have been identified to be significantly associated with $\mathrm{PH}$, and QTLs on chromosomes $1 \mathrm{H}, 2 \mathrm{H}, 5 \mathrm{H}$, and $6 \mathrm{H}$ were shown to be significantly associated with SL in spring barley exposed to drought (Fakheri et al., 2018). In our study, SNPs that are significantly associated with $\mathrm{HD}$ and $\mathrm{PH}$ were identified on almost all barley chromosomes, and the SNPs mainly identified on chromosomes $1 \mathrm{H}, 2 \mathrm{H}, 3 \mathrm{H}, 4 \mathrm{H}$, and $7 \mathrm{H}$ had significant effects on SL. To validate the effect estimation of each QTL, QTL effect estimations were used to predict the observed phenotypic performance. The highest prediction accuracy was $69.2 \%$ for $\mathrm{HD}$, and the lowest prediction accuracy was $23.55 \%$ for SL in Namling. In the present study, heritability for all traits ranged from 44.97 and $73.99 \%$. For these traits, both the number of detected QTL, prediction accuracy, and broad-sense heritability showed the same trend in which higher heritability corresponded to high prediction accuracy and more detected QTLs. 
In order to figure out the specific QTLs for qingke barley, QTLs reported in the reference and identified in this study were aligned to the barley Hordeum vulgare Hv IBSC PGSB v2 reference genome, and their physical positions of markers were queried in BARLEX database (https://apex.ipk-gatersleben. de/apex/f?p=284:48:::NO:RP:P48_MARKER_CHOICE:4). As a result, 48 of 62 identified QTLs were first reported in this study (Table 2 and Supplementary Figure 13). For HD, 36 QTLs were identified, 7 of which were reported; for $\mathrm{PH}$, 33 QTLs were identified, 9 of which were reported; and for SL, 22 QTLs were identified, 5 of which were reported. The possible reason for the high number of novel QTLs (viewed as qingke barley specific QTLs) identified in this study may be because (1) the genetics of qingke barley is lack of analysis; and (2) some reported QTLs based on SSR markers could not find the their physical positions, and some reported QTLs developed by in-house SNP chips could not match the chip version in the database. These qingke barley specific QTLs could be utilized for marker-assisted selection in qingke barley breeding programs focusing on adaption and high grain yield.

Among the five common vernalization and photoperiod loci (i.e., $H v V R N 1, H v V R N 2, H v V R N 3, P p d-H 1$, and $P p d-H 2)$, the SNPs near $H v V R N 1, H v V R N 3$, and $P p D-H 2$ were significant in this study. It suggests that $H v V R N 1, H v V R N 3$, and $P p d-H 2$ play an important role in the qingke barley population, and should be prioritized when attempting to improve HD and plant growth in qingke barley cultivars in the qingke barleygrowing regions of the Qinghai-Tibet Plateau. Previous studies have shown that QTLs for PH and SL are located on different chromosomes depending on the different environments and treatments (Gyenis et al., 2007; Fakheri et al., 2018). In the present study, we observed QTLs related to $\mathrm{PH}$ on all chromosomes, while QTLs associated with SL were detected on all chromosomes in Namling but on $1 \mathrm{H}, 2 \mathrm{H}, 3 \mathrm{H}, 4 \mathrm{H}$, and $7 \mathrm{H}$ in Nyingchi. Due to the differences in locations of QTLs identified in different environments, the expression of genes controlling $\mathrm{HD}, \mathrm{PH}$, and SL are probably related to the environment and varietyspecific adaptability. To validate this hypothesis, the study of selection signals in the qingke barley genome were conducted to help us understand how qingke barley how qingke barley responds to various historical environmental factors (Russell et al., 2016). Eight regions were identified as candidate selective regions, and these are distributed on all chromosomes except for chromosome $4 \mathrm{H}$ (Zeng et al., 2018). We used the first 10 eigenvectors for EigenGWAS and identified several selected loci in the qingke barley genome. We further compared the selected loci with the located QTLs, and found that some of these loci were located in the regions $(1 \mathrm{Mb})$ of these QTLs (Table 1). Previous studies have shown that genes for $\mathrm{HD}$ and $\mathrm{PH}$ always influence barley maturity and adaptation (Barua et al., 1993; Laurie et al., 1994). In the present study, the results of EigenGWAS analysis indicated that the QTLs associated with HD and $\mathrm{PH}$ also bear signatures of genetic selection in this qingke barley population.

\section{CONCLUSION}

In this study, we identified several genetic loci associated with $\mathrm{SL}, \mathrm{PH}$, and $\mathrm{HD}$ in qingke barley from the Qinghai-Tibet Plateau using 14,970 SNPs in a tGBS genotyping assay. We found that more rare SNPs $(2,150)$ were found in the landrace subpopulation than in the variety subpopulation. That is to say, the number of SNPs was higher in the varieties than in the landraces, indicating that the varieties grown in Tibet and the varieties from around the Tibetan area may be derived from the different landraces grown in the different regions. A GWAS identified 62 QTLs that are associated with $\mathrm{HD}, \mathrm{PH}$, and SL, and 114 known genes were mapped which include, but are not limited to, genes involved in vernalization and photoperiod. Of the 62 QTLs, 48 are first reported here as qingke specific QTLs, $52(83.87 \%)$ were found to be in intergenic regions, 28 had pleotropic effects, and three QTL were in the regions of the well-characterized genes $H v V R N 1, H v V R N 3$, and PpD-H2. In addition, by comparing signatures of selection identified by EigenGWAS and novel QTLs, we found that six QTLs related to $\mathrm{HD}$ and $\mathrm{PH}$ in qingke barley cultivars from the QinghaiTibet Plateau were also under selection. The findings presented here could help increase our understanding of the genetic mechanisms underlying the establishment of adaptive traits, and also enable marker-assisted selection for important traits in qingke barley breeding.

\section{DATA AVAILABILITY STATEMENT}

The datasets generated for this study can be found in the https:// www.ncbi.nlm.nih.gov/sra/PRJNA606408.

\section{AUTHOR CONTRIBUTIONS}

This study was designed by HL and DD. The experiment was performed under the support of GG, JZ, and TN. The evaluation of traits was conducted by NL, KD, PC, LG, and WC. Data were analyzed by ZL, NL, and HL. The manuscript was drafted by ZL, NL, and HL, and revised by GG and JW. All authors contributed to the article and approved the submitted version.

\section{FUNDING}

This study was supported by the National Natural Science Foundation of China (31660299), the Tibet Department of Key Projects (XZ201801NA01), and the National Key Research and Development Program of China (2015BAD02B01-2-2).

\section{SUPPLEMENTARY MATERIAL}

The Supplementary Material for this article can be found online at: https:/www.frontiersin.org/articles/10.3389/fgene. 2020.00638/full\#supplementary-material 


\section{REFERENCES}

Abdullaev, R. A., Alpatieva, N. V., Zveinek, I. A., Batasheva, B. A., Anisimova, I. M., and Radchenko, E. E. (2017). Diversity of dagestan barleys for the duration of the period between shooting and earing stages and alleles in the Ppd-H1 and Ppd-H2 loci. Russ. Agric. Sci. 43, 99-103. doi: 10.3103/ S1068367417020021

Almerekova, S., Sariev, B., Abugalieva, A., Chudinov, V., Sereda, G., Tokhetova, L., et al. (2019). Association mapping for agronomic traits in six-rowed spring barley from the USA harvested in Kazakhstan. PLoS ONE 14:e0221064. doi: 10.1371/journal.pone.0221064

Alqudah, A. M., Koppolu, R., Wolde, G. M., Graner, A., and Schnurbusch, T. (2016). The genetic architecture of barley plant stature. Front. Genet. 7:117. doi: 10.3389/fgene.2016.00117

Alqudah, A. M., Shama, R., Pasam, R. K., Graner, A., Kilian, B., et al. (2014). Genetic dissection of photoperiod response based on GWAS of pre-anthesis phase duration in spring barley. PLoS ONE 9:e113120. doi: 10.1371/journal.pone. 0113120

Alqudah, A. M., Youssef, H. M., Graner, A., and Schnurbusch, T. (2018). Natural variation and genetic make-up of leaf blade area in spring barley. Theor. Appl. Genet. 131, 873-886. doi: 10.1007/s00122-018-3053-2

Al-Tabbal, J. A., and Al-Fraihat, A. H. (2012). Genetic variation, heritability, phenotypic and genotypic correlation studies for yield and yield components in promising barley genotypes. J. Agric. Sci. 4:193. doi: 10.5539/ jas.v4n3p193

Badr, A., Muller, K., Sch, R., Rabey, H. E., Effgen, S., Ibrahim, H. H., et al. (2000). On the origin and domestication history of barley (Hordeum vulgare). Mol. Biol. Evol. 17, 499-510. doi: 10.1093/oxfordjournals.molbev.a026330

Barua, U. M., Chalmers, K. J., Thomas, W. T. B., Hackett, C. A., Lea, V., Jack, P., et al. (1993). Molecular mapping of genes determining height, time to heading, and growth habit in barley (Hordeum vulgare). Genome 36, 1080-1087. doi: $10.1139 / \mathrm{g} 93-143$

Beales, J., Turner, A., Griffiths, S., Snape, J. W., and Laurie, D. A. (2007). A pseudoresponse regulator is misexpressed in the photoperiod insensitive Ppd-D1a mutant of wheat (Triticum aestivum L.). Theor. Appl. Genet. 115, 721-733. doi: $10.1007 / \mathrm{s} 00122-007-0603-4$

Blake, T., Blake, V. C., Bowman, J. G. P., and Abdel-Haleem, H. (2010). Barley feed uses and quality improvement. Barley 522-531. doi: 10.1002/9780470958636.ch16

Bradbury, P. J., Zhang, Z., Kroon, D. E., Casstevents, T. M., Ramdoss, Y., and Buckler, E. S. (2007). TASSEL: software for association mapping of complex traits in diverse samples. Bioinformatics 23, 2633-2635. doi: 10.1093/bioinformatics/btm308

Cai, S., Wu, D., Jabeen, Z., Huang, Y. Q., Huang, Y. C., and Zhang, G. P. (2013). Genome-wide association analysis of aluminum tolerance in cultivated and Tibetan wild barley. PLoS ONE 8:e69776. doi: 10.1371/journal.pone.0069776

Chen, G. B., Lee, S. H., Zhu, Z. X., and Robinson, M. R. (2016). EigenGWAS: finding loci under selection through genome-wide association studies of eigenvectors in structured populations. Heredity 117:51. doi: 10.1038/hdy.2016.25

Chono, M., Honda, I., Zeniya, H., Yoneyama, Z., Saisho, D., Takeda, K., et al. (2003). A semidwarf phenotype of barley uzu results from a nucleotide substitution in the gene encoding a putative brassinosteroid receptor. Plant Physiol. 133, 1209-12219. doi: 10.1104/pp.103.026195

Cingolani, P., Platts, A., Wang, L. L., Coon, M., Nguyen, T., Wang, L., et al. (2012). A program for annotating and predicting the effects of single nucleotide polymorphisms, SnpEff: SNPs in the genome of Drosophila melanogaster strain w1118; iso-2; iso-3. Fly 6, 80-92. doi: 10.4161/fly.19695

Collins, H. M., Burton, R. A., Topping, D. L., Liao, M. L., Bacic, A., and Fincher, G. B. (2010). Variability in fine structures of noncellulosic cell wall polysaccharides from cereal grains: potential importance in human health and nutrition. Cereal. Chem. 87, 272-282. doi: 10.1094/CCHEM-87-4-0272

Colmsee, C., Beier, S., Himmelbach, A., Schmutzer, T., Stein, N., Scholz, U., et al. (2015). BARLEX-the barley draft genome explorer. Mol. Plant. 8, 964-966. doi: 10.1016/j.molp.2015.03.009

Comadran, J., Russell, J. R., Booth, A., Pswarayi, A., Ceccarelli, S., Grando, S., et al. (2011). Mixed model association scans of multi-environmental trial data reveal major loci controlling yield and yield related traits in Hordeum vulgare in Mediterranean environments. Theor. Appl. Genet. 122, 1363-1373. doi: 10.1007/s00122-011-1537-4

Cuesta-Marcos, A., Igartua, E., Codesal, P., Ciudad, F. J., Codesal, P., Russell, J. R., et al. (2008). Heading date QTL in a spring $\times$ winter barley cross evaluated in Mediterranean environments. Mol. Breed. 21, 455-471. doi: 10.1007/s11032-007-9145-3

Dai, F., Chen, Z. H., Wang, X., Li, Z., Jin, G., Wu, D., et al. (2014). Transcriptome profiling reveals mosaic genomic origins of modern cultivated barley. Proc. Natl. Acad. Sci. U.S.A. 111, 13403-13408. doi: 10.1073/pnas.1414 335111

Dai, F., Nevo, E., Wu, D., Comadran, J., Zhou, M., Qiu, L., et al. (2012). Tibet is one of the centers of domestication of cultivated barley. Proc. Natl. Acad. Sci. U.S.A. 109, 16969-16973. doi: 10.1073/pnas.1215265109

Devlin, B., and Roeder, K. (1999). Genomic control for association studies. Biometrics 55, 997-1004. doi: 10.1111/j.0006-341X.1999.00997.x

Distelfeld, A., Li, C., and Dubcovsky, J. (2009). Regulation of flowering in temperate cereals. Curr. Opin. Plant Biol. 12, 178-184. doi: $10.1016 /$ j.pbi.2008.12.010

Dockter, C., and Hansson, M. (2015). Improving barley culm robustness for secured crop yield in a changing climate. J. Exp. Bot. 3499-3509. doi: 10.1093/jxb/eru521

Fakheri, B. A., Aghnoum, R., Nezhad, N. M., and Ataei, R. (2018). GWAS analysis in spring barley (Hordeum vulgare L.) for morphological traits exposed to drought. PLoS ONE 13:e0204952. doi: 10.1371/journal.pone.0204952

Froster, B. P. (2001). Mutation genetics of salt tolerance in barley: an assessment of Golden Promise and other semi-dwarf mutants. Euphytica. 120, 317-328. doi: 10.1023/A:1017592618298

Genievskaya, Y., Almerekova, S., Sariev, B., Chudinov, V., Tokhetova, L., Sereda, G., et al. (2018). Marker-trait associations in two-rowed spring barley accessions from Kazakhstan and the USA. PLoS ONE 13:e0205421. doi: 10.1371/journal.pone.0205421

Gyawali, S., Otte, M. L., Chao, S., Jilal, A., Jacob, D. L., Amezrou, R., et al. (2017). Genome wide association studies (GWAS) of element contents in grain with a special focus on zinc and iron in a world collection of barley (Hordeum vulgare L.). J. Cereal Sci. 77, 266-274. doi: 10.1016/j.jcs.2017.08.019

Gyenis, L., Yun, S. J., Smith, K. P., Steffenson, B. J., Bossolini, E., Sanguineti, M. C., et al. (2007). Genetic architecture of quantitative trait loci associated with morphological and agronomic trait differences in a wild by cultivated barley cross. Genome 50, 714-723. doi: 10.1139/G07-054

Hemming, M. N., Fieg, S., Peacock, W. J., and Dennis, E. S., Trevaskis, B. (2009). Regions associated with repression of the barley (Hordeum vulgare) VERNALIZATION1 gene are not required for cold induction. Mol. Genet. Genomics 282, 107-117. doi: 10.1007/s00438-009-0449-3

Hill, C. B., Angessa, T. T., McFawn, L. A., Wong, D., Tibbits, J., Zhang, X. Q., et al. (2019). Hybridisation-based target enrichment of phenology genes to dissect the genetic basis of yield and adaptation in barley. Plant Biotechnol. J. 17, 932-944. doi :10.1111/pbi.13029 doi: 10.1111/pbi.13029

Hu, X., Zuo, J., Wang, J., Liu, L., Sun, G., Li, C., et al. (2018). Multi-locus genomewide association studies for 14 Main agronomic traits in barley. Front. Plant Sci. 9:1683. doi: 10.3389/fpls.2018.01683

Ingvordsen, C. H., Backes, G., Lyngkjær, M. F., Peltonen-Sainio, P., Jensen, J. D., Jalli, M., et al. (2015). GWAS of barley phenotypes established under future climate conditions of elevated temperature, $\mathrm{CO}_{2}, \mathrm{O}_{3}$ and elevated temperature and $\mathrm{CO}_{2}$ combined. Proc. Environ. Sci. 29, 164-165. doi: 10.1016/j.proenv.2015.07.241

Jia, Q. J., Zhang, J. J., Westcott, S., Zhang, X. Q., Bellgard, M., Lance, R., et al. (2009). GA-20 oxidase as a candidate for the semidwarf gene sdw1/denso in barley. Funct. Integr. Genomics. 9, 255-262. doi: 10.1007/ s10142-009-0120-4

Kiseleva, A. A., Shcherban, A. B., Leonova, I. N., Frenkel, Z., and Salina, E. A. (2016). Identification of new heading date determinants in wheat $5 B$ chromosome. BMC Plant Biol. 16:8. doi: 10.1186/s12870-015-0688-x

Kuczynska, A., Surma, M., Adamski, T., Mikołajczak, K., Krystkowiak, K., and Ogrodowicz, P. (2013). Effects of the semi-dwarfing sdw1/denso gene in barley. Plant Genet. 54, 381-390. doi: 10.1007/s13353-013-0165-x

Laurie, D. A., Pratchett, N., Bezant, J. H., and Snape, J. W. (1994). Genetic analysis of a photoperiod response gene on the short arm of chromosome $2(2 \mathrm{H})$ of Hordeum vulgare (barley). Heredity 72:619. doi: 10.1038/hdy.1994.85 
Laurie, D. A., Pratchett, N., Snape, J. W., and Bezant, J. H. (1995). RFLP mapping of five major genes and eight quantitative trait loci controlling flowering time in a winter $\times$ spring barley (Hordeum vulgare L.) cross. Genome 38, 575-585. doi: $10.1139 / \mathrm{g} 95-074$

Li, J., Chen, G. B., Rasheed, A., Li, D., Sonder, K., Zavala Espinosa, C., et al. (2019). Identifying loci with breeding potential across temperate and tropical adaptation via EigenGWAS and EnvGWAS. Mol. Ecol. 28, 3544-3560. doi: $10.1111 / \mathrm{mec} .15169$

Li, Q., Pan, Z., Deng, G., Long, H., Li, Z., Deng, X., et al. (2014). Effect of wide variation of the waxy gene on starch properties in hull-less barley from Qinghai-Tibet Plateau in China. J. Agric. Food Chem. 62, 11369-11385. doi: $10.1021 /$ jf5026746

Li, S., Wang, J., and Zhang, L. (2015). Inclusive composite interval mapping of QTL by environment interactions in biparental populations. PLOS ONE 10:e0132414. doi: 10.1371/journal.pone.0132414

Lin, S. Y., Sasaki, T., and Yano, M. (1998). Mapping quantitative trait loci controlling seed dormancy and heading date in rice, Oryza sativa L., using backcross inbred lines. Theor. Appl. Genet. 96, 997-1003. doi: $10.1007 /$ s001220050831

Mascher, M., Gundlach, H., Himmelbach, A., Beier, S., Twardziok, S. O., Wicker, T., et al. (2017). A chromosome conformation capture ordered sequence of the barley genome. Nature 544, 427-433. doi: 10.1038/nature22043

Mei, W., Stetter, M. G., Gates, D. J., Stitzer, M., and Ross-Ibarra, J. (2017). Adaptation in plant genomes: bigger isn't better, but it's probably different. Am. J. Bot. 105, 16-19. doi: 10.1101/196501

Meng, L., Li, H., Zhang, L., and Wang, J. (2015). QTL IciMapping: integrated software for genetic linkage map construction and quantitative trait locus mapping in biparental populations. Crop J. 3, 269-283. doi: 10.1016/j.cj.2015.01.001

Mikołajczak, K., Kuczynska, A., Krajewski, P., Sawikowska, A., Surma, M., Piotr, O., et al. (2017). Quantitative trait loci for plant height in Maresi $\times$ CamB barley population and their associations with yield-related traits under different water regimes. J. Appl. Genet. 58, 23-35. doi: 10.1007/s13353-016-0358-1

Ott, A., Liu, S., Schnable, J. C., Yeh, C. T. E., Wang, K. S., and Schnable, P. S. (2017). $\mathrm{tGBS}^{\circledR}$ genotyping-by-sequencing enables reliable genotyping of heterozygous loci. Nucleic Acids Res. 45:e178. doi: 10.1093/nar/gkx853

Paradis, E., Claude, J., and Strimmer, K. (2004). APE: analyses of phylogenetics and evolution in R language. Bioinformatics. 20, 289-290. doi: 10.1093/bioinformatics/btg412

Pasam, R. K., Sharma, R., Malosetti, M., Eeuwijk, F. A. V., Haseneyer, G., Kilian, B., et al. (2012). Genome-wide association studies for agronomical traits in a worldwide spring barley collection. BMC Plant Biol. 12:16. doi: 10.1186/1471-2229-12-16

Pauli, D., Muehlbauer, G. J., Smith, K. P., Cooper, B., Hole, D., Obert, D. E., et al. (2014). Association mapping of agronomic QTLs in US spring barley breeding germplasm. Plant Genome 7, 1-15. doi: 10.3835/plantgenome2013.11.0037

Pham, A. T., Maurer, A., Pillen, K., Brien, C., Dowling, K., Berger, B., et al. (2019). Genome-wide association of barley plant growth under drought stress using a nested association mapping population. BMC Plant Biol. 19:134. doi: 10.1186/s12870-019-1723-0

Powell, J. E., Visscher, P. M., and Goddard, M. E. (2010). Reconciling the analysis of IBD and IBS in complex trait studies. Nat. Rev. Genet. 11, 800-805. doi: $10.1038 / \mathrm{nrg} 2865$

Qiu, L., Wu, D., Ali, S., Cai, S., Dai, F., Jin, X., et al. (2011). Evaluation of salinity tolerance and analysis of allelic function of HvHKT1 and HvHKT2 in Tibetan wild barley. Theor. Appl. Genet. 122, 695-703. doi: 10.1007/ s00122-010-1479-2

Remington, D. L., Thornsberry, J. M., Matsuoka, Y., Wilson, L. M., Whitt, S. R., Doebley, J., et al. (2001). Structure of linkage disequilibrium and phenotypic associations in the maize genome. Proc. Natl. Acad. Sci. U.S.A. 98, 11479-11484. doi: $10.1073 /$ pnas. 201394398

Russell, J., Mascher, M., Dawson, I. K., Kyriakidis, S., Calixto, C., Freund, F., et al. (2016). Exome sequencing of geographically diverse barley landraces and wild relatives gives insights into environmental adaptation. Nat. Genet. 48:1024. doi: $10.1038 /$ ng.3612

Saitou, N., and Nei, M. (1987). The neighbor-joining method: a new method for reconstructing phylogenetic trees. Mol. Biol. Evol. 4, 406-425.
Sameri, M., Takeda, K., and Komatsuda, T. (2006). Quantitative trait loci controlling agronomic traits in recombinant inbred lines from a cross of oriental-and occidental-type barley cultivars. Breed. Sci. 56, 243-252. doi: 10.1270/jsbbs.56.243

Tashi, N., Yawei, T., and Xingquan, Z. (2013). "Food preparation from hulless barley in Tibet," in Advance In Barley Sciences, eds G. Zhang, C. Li, and X. Liu (Dordrecht: Springer), 151-158.

Tian, T., Liu, Y., Yan, H., You, Q., Yi, X., Du, Z., et al. (2017). agriGO v2. 0: a GO analysis toolkit for the agricultural community, 2017 update. Nucleic Acids Res. 45, W122-W129. doi: 10.1093/nar/gkx382

Tondelli, A., Xu, X., Moragues, M., Sharma, R., Schnaithmann, F., Ingvardsen, C., et al. (2013). Structural and temporal variation in genetic diversity of european spring two-row barley cultivars and association mapping of quantitative traits. Plant Genome 6, 1-14. doi: 10.3835/plantgenome2013. 03.0007

Trevaskis, B. (2010). The central role of the VERNALIZATION1 gene in the vernalization response of cereals. Funct. Plant Biol. 37, 479-487. doi: 10.1071/FP10056

Trevaskis, B., Bagnall, D. J., Ellis, M. H., Peacock, W. J., and Dennis, E. S. (2003). MADS box genes control vernalization-induced flowering in cereals. Proc. Natl. Acad. Sci. U.S.A. 100, 13099-13104. doi: 10.1073/pnas.1635053100

Trevaskis, B., Hemming, M. N., Peacock, W. J., and Dennis, E. S. (2006). HvVRN2 responds to daylength, whereas HvVRN1 is regulated by vernalization and developmental status. Plant Physiol. 140, 1397-1405. doi: 10.1104/pp.105.073486

Turner, A., Beales, J., Faure, S., Faure, S., Dunford, R. P., and Laurie, D. A. (2005). The pseudo-response regulator Ppd-H1 provides adaptation to photoperiod in barley. Science 310, 1031-1034. doi: 10.1126/science.1117619

Ullrich, S. E. (2010). Barley: Production, Improvement, and Uses,Vol. 12. New Jersey, NJ: John Wiley \& Sons.

Visioni, A., Tondelli, A., Francia, E., Pswarayi, A., Malosetti, M., Russell, J., et al. (2013). Genome-wide association mapping of frost tolerance in barley (Hordeum vulgare L.). BMC Genomics 14:424. doi: 10.1186/ 1471-2164-14-424

Visioni, A. V., Gyawali, S., Selvakumar, R., Gangwar, O. P., Shekhawat, P. S., and Bhardwaj, S. C. (2018). Genome wide association mapping of seedling and adult plant resistance to barley stripe rust (Puccinia striiformis f. sp. hordei) in India. Front. Plant Sci. 9:520. doi: 10.3389/fpls.2018.00520

Wang, J., Yang, J., McNeil, D. L., and Zhou, M. (2010). Identification and molecular mapping of a dwarfing gene in barley (Hordeum vulgare L.) and its correlation with other agronomic traits. Euphytica 175, 331-342. doi: 10.1007/s10681-010-0175-2

Wang, J. B., Sun, G., Ren, X. F., Li, C. D., Liu, L. P., Wang, Q. F., et al. (2016). QTL underlying some agronomic traits in barley detected by SNP markers. BMC Genet. 17:103. doi: 10.1186/s12863-016-0409-y

Weir, B. S., and Cockerham, C. (1996). Genetic Data Analysis II: Methods for Discrete Population Genetic Data. Sunderland, MA: Sinauer Associates, Inc.

Wu, D., Qiu, L., Xu, L., Ye, L., Chen, M., Sun, D., et al. (2011). Genetic variation of $\mathrm{HvCBF}$ genes and their association with salinity tolerance in Tibetan annual wild barley. PLOS ONE 6:e22938. doi: 10.1371/journal.pone. 0022938

Xu, X., Sharma, R., Tondelli, A., Russell, J., Comadran, J., Schnaithmann, F., et al. (2018). Genome-wide association analysis of grain yield-associated traits in a pan-European barley cultivar collection. Plant Genome 11:170073. doi: 10.3835/plantgenome2017.08.0073

Xu, Y. H., Jia, Q. J., Zhou, G. F., Zhang, X. Q., Angessa, T., Broughton, S., et al. (2017). Characterization of the sdw1 semi-dwarf gene in barley. BMC Plant Biol. 17:11. doi: 10.1186/s12870-016-0964-4

Yan, L., Fu, D., Li, C., Blechi, A., Tranquilli, G., Bonafede, M., et al. (2006). The wheat and barley vernalization gene VRN3 is an orthologue of FT. Proc. Natl. Acad. Sci. U.S.A. 103, 19581-19586. doi: 10.1073/pnas. 0607142103

Yan, L., Helguera, M., Kato, K., Fukuyama, S., Sherman, J., and Dubcovsky, J. (2004). Allelic variation at the VRN-1 promoter region in polyploid wheat. Theor. Appl. Genet. 109, 1677-1686. doi: 10.1007/s00122004-1796-4 
Yan, L., Loukoianov, A., Tranquilli, G., Helguera, M., Fahima, T., and Dubcovsky, J. (2003). Positional cloning of the wheat vernalization gene VRN1. Proc. Natl. Acad. Sci. U.S.A. 100, 6263-6268. doi: 10.1073/pnas.0937399100

Yan, W. H., Wang, P., Chen, H. X., Zhou, H. J., Li, Q. P., and Wang, C. R. (2011). A major QTL, Ghd8, plays pleiotropic roles in regulating grain productivity, plant height, and heading date in rice. Mol. Plant. 4, 319-330. doi: 10.1093/mp/ssq070

Zeng, X., Bai, L., Wei, Z., Yuan, H., Wang, Y., Xu, Q., et al. (2016). Transcriptome analysis revealed the drought-responsive genes in Tibetan hulless barley. BMC Genomics 17:386. doi: 10.1186/s12864-016-2685-3

Zeng, X., Guo, Y., Xu, Q., Mascher, M., Guo, G., Li, S., et al. (2018). Origin and evolution of qingke barley in Tibet. Nat. Commun. 9:5433. doi: 10.1038/s41467-018-07920-5

Zeng, X., Long, H., Wang, Z., Zhao, S., Tang, Y., Huang, Z., et al. (2015). The draft genome of Tibetan hulless barley reveals adaptive patterns to the high stressful Tibetan Plateau. Proc. Natl. Acad. Sci. U.S.A. 112, 1095-1100. doi: $10.1073 /$ pnas. 1423628112

Zhang, J. (2000). Inheritance of agronomic traits from the Chinese barley dwarfing gene donors 'Xiaoshan Lixiahuang' and 'Cangzhou Luodama'. Plant Breed. 119, 523-524. doi: 10.1046/j.1439-0523.2000.00543.x

Zhang, K., Tian, J., Zhao, L., Liu, B., and Chen, G. (2009). Detection of quantitative trait loci for heading date based on the doubled haploid progeny of two elite Chinese wheat cultivars. Genetica 135, 257-265. doi: 10.1007/s10709-008-9274-6
Zhang, M., Fu, M. M., Qiu, C. W., Cao, F., Chen, Z. H., Zhang, G., et al. (2019). Response of tibetan wild barley genotypes to drought stress and identification of quantitative trait loci by genome-wide association analysis. Int. J. Mol. Sci. 20:791. doi: 10.3390/ijms20030791

Zhang, Z., Ersoz, E., Lai, C. Q., Todhunter, R. J., Tiwari, H. K., Gore, M. A., et al. (2010). Mixed linear model approach adapted for genome-wide association studies. Nat. Genet. 42:355. doi: 10.1038/ng.546

Zhou, H., and Steffenson, B. (2013). Genome-wide association mapping reveals genetic architecture of durable spot blotch resistance in US barley breeding germplasm. Mol. Breed. 32, 139-154. doi: 10.1007/ s11032-013-9858-4

Conflict of Interest: The authors declare that the research was conducted in the absence of any commercial or financial relationships that could be construed as a potential conflict of interest.

Copyright (c) 2020 Li, Lhundrup, Guo, Dol, Chen, Gao, Chemi, Zhang, Wang, Nyema, Dawa and Li. This is an open-access article distributed under the terms of the Creative Commons Attribution License (CC BY). The use, distribution or reproduction in other forums is permitted, provided the original author(s) and the copyright owner(s) are credited and that the original publication in this journal is cited, in accordance with accepted academic practice. No use, distribution or reproduction is permitted which does not comply with these terms. 